\title{
Modelling of Target-Controlled Infusion of Propofol for Depth-of-Anaesthesia Simulation in Matlab-Simulink
}

\author{
Gorazd Karer \\ Faculty of Electrical Engineering, University of Ljubljana, Slovenia, gorazd.karer@fe.uni-lj.si
}

\begin{abstract}
Total intravenous anaesthesia (TIVA) is an anaesthesiologic technique, where substances are injected intravenously. The anaesthesiologist adjusts the injection of intravenous anaesthetic agents regarding the depth of anaesthesia. In the paper, we present a model of an anaesthetic agent, namely propofol, influencing the depth of anaesthesia. The influence of propofol is linked to the concentration of the drug in the appropriate compartment. First, the modelling of pharmacokinetics of propofol is introduced. The 3-compartmental model and the effect-site model are presented, the relevant model parameters are given. Next, the model is verified by comparing the simulation results to the data file that was recorded by the Orchestra Base Primea infusion workstation during a medical procedure, which lasted about 40 minutes. The simulation results are presented and the predictive quality of the model is evaluated.
\end{abstract}

The presented model for Matlab-Simulink provides a basic tool for further researching the dynamics of anaesthetic depth. Despite the fact that more data must be obtained in order to properly validate the model, the presented model provides a basis for running simulations and testing various scenarios of propofol administration and is usable for developing and testing closed-loop control approaches for automatic control of depth of anaesthesia.

Keywords: target-controlled infusion, Propofol, depthof-anaesthesia, Matlab-Simulink

\section{Introduction}

To perform a general anaesthesia, it is necessary to use substances, which enable deep unconsciousness, analgesia, amnesia and muscle relaxation, all required for performing a surgery or a diagnostic procedure. General anaesthesia and related dynamic activities in the human body is a complicated process, which includes pharmacokinetic and pharmacodynamic mechanisms, which have not been fully studied yet.

During the general anaesthesia, the anaesthesiologist needs to monitor the patient's vital functions and maintain the functions of vital organs. To achieve anaesthesia, substances are introduced in different manners into the patient's body. In clinical practice, the most commonly used methods are the intravenous induction of an anaesthetic agent, i.e., injection of the anaesthetic into a vein, and in- halation induction of anaesthesia, whereby the patient inhales the substance from the breathing mixture. Total intravenous anaesthesia (TIVA) is an anaesthesiologic technique, where substances are injected intravenously.

The anaesthesiologist needs to adjust the dosage of anaesthetic to maintain the appropriate depth of general anaesthesia according to pharmacokinetics and pharmacodynamics of the anaesthetic agent and considering the type of procedure. Inadequate depth of anaesthesia is manifested with the activation of sympathetic nerves or in the most unlikely event with the patient awakening. Too deep anaesthesia is manifested with a drop in blood pressure level and heart rate frequency as well as slow postoperative awakening of the patient from general anaesthesia. In modern clinical practice, the depth of anaesthesia is determined by assessing the relevant clinical signs (iris, sweating, movements), by interpreting hemodynamic measurements (Potočnik et al., 2011) and by estimating the depth of anaesthesia from EEG signals, for which several established measurement systems already exist, e.g. BIS index, Narcotrend, Scale Entropy and Response Entropy. BIS index measurement is a non-invasive method, where a BIS monitor is connected to electrodes on the patient's head. By measuring the EEG signals the bispectral index is defined, representing the depth of anaesthesia. The BIS monitor provides a single dimensionless number, which ranges from 0 (equivalent to EEG silence) to 100. A BIS value between 40 and 60 indicates an appropriate level for general anaesthesia, whereas for long-term sedation due to head injuries a value below 40 is appropriate. The reference can thus be set to the applicable value; the manner and speed of approaching the reference value depend on the specific characteristics of the procedure and the pharmacokinetics and pharmacodynamics of the substance in the patient's body.

The problem of modelling the effect of propofol is described in literature in various ways. For such purposes, pharmacokinetic and pharmacodynamic models have been developed, such as in (Marsh et al., 1991; Schnider et al., 1998, 1999; Kataria et al., 1994; Schüttler and Ihmsen, 2000; Kenny and White, 1990). The models typically define the basic structure of the dynamic operating system of propofol and the parameters depend on individual patients. The values of model's parameters are affected by the patient and his characteristics (weight, height, age, sex etc.) as well as individual sensitivity to propofol and the 
ability to excrete propofol.

Several developed pharmacokinetic models are used in certain infusion pumps for target controlled infusion (TCI), where the pump sets the proper flow of the medication with regard to the model. The problem with these models is that they often do not reflect the real dynamics, which also depends on individual sensitivity of the patients to the substance, therefore such approaches, based on open-loop induction, often do not yield the best performance.

In the paper, we present a dynamical model of propofol influencing the depth of anaesthesia, which is connected to the concentration of the drug in the appropriate compartments. The paper is organized as follows. First, modelling of pharmacokinetics of propofol is introduced. The 3-compartmental model and the effect-site model are presented, the relevant model parameters are given. Next, the model is verified by comparing the simulation results to the data file of an actual anaesthetic application of targetcontrolled infusion of intravenously administered propofol, which was recorded by the Orchestra Base Primea infusion workstation during a medical procedure that lasted about 40 minutes. The simulation results are presented and the predictive quality of the model is evaluated. Finally, we give some concluding remarks.

\section{Modelling the pharmacokinetics of propofol}

\subsection{The 3-compartmental model}

The pharmacokinetics of the derived model is based on the Marsh model (Marsh et al., 1991). The dynamic relations are based on a well-established 3-compartmental model structure, as shown in Figure 1.

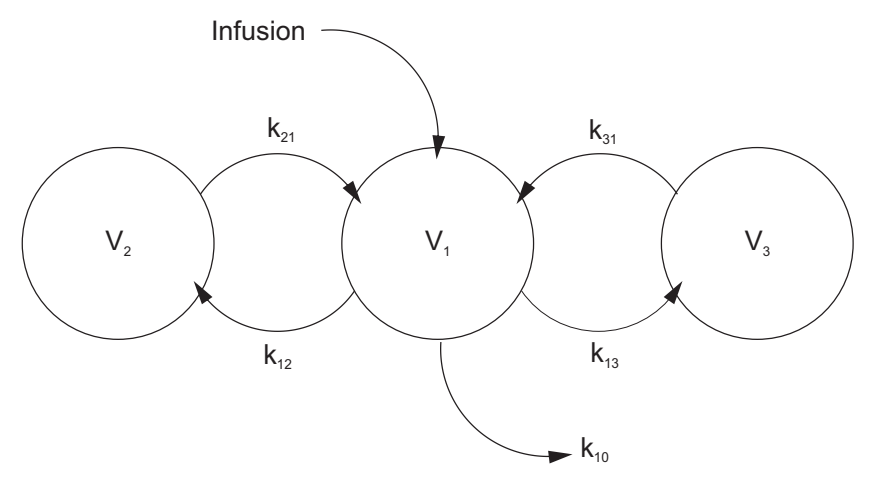

Figure 1. The pharmacokinetics of the 3-compartmental model.

The 3-compartmental model can be described as follows:

- The drug (namely propofol) is injected intravenously into the central compartment $\left(V_{1}\right)$, representing the blood (or plasma) in the body - contained primarily in the arteries and veins and the directly influenced tissues and organs, such as brain, heart, liver, kidney etc.
- The second compartment $\left(V_{2}\right)$ represents the group of tissues that are indirectly affected by the amount of drug in the central compartment, i.e., mainly the muscles. The exchange of the drug with the central compartment is denoted by $k_{12}$ and $k_{21}$.

- The third compartment $\left(V_{3}\right)$ represents the group of tissues that can store a certain amount of drug, but the exchange with the central compartment is rather slow, i.e., mainly the fat. However, the amount of drug in these tissues influences the amount of the drug in the central compartment in the long run. The exchange of the drug with the central compartment is denoted by $k_{13}$ and $k_{31}$.

- The drug is eliminated from the body with a rate denoted by $k_{10}$.

The internal dynamics of the model can be formulated by using

$$
\begin{gathered}
\frac{d x_{1}}{d t}=\phi-k_{12} x_{1}-k_{13} x_{1}-k_{10} x_{1}+k_{21} x_{2}+k_{31} x_{3} \\
\frac{d x_{2}}{d t}=-k_{21} x_{2}+k_{12} x_{1} \\
\frac{d x_{3}}{d t}=-k_{31} x_{3}+k_{13} x_{1}
\end{gathered}
$$

where the variables $x_{1}, x_{2}$, and $x_{3}$ represent the amount of the drug in compartment $V_{1}, V_{2}$, and $V_{3}$. respectively. The infusion flow rate is denoted as $\phi$. As noted above, the parameters $k_{12}, k_{21}, k_{13}$, and $k_{31}$, represent the partition coefficients that determine the speed at which the drug goes from one particular compartment to another. Finally, $k_{10}$ is the rate of elimination of the drug from the body.

Note that the concentration in the central compartment is often referred to as plasmatic concentration.

\subsection{Effect-site concentration model}

The effect site for the drug propofol is basically the central nervous system. The effect site is thus part of the central compartment, but the effect of the drug is subject to some dynamics with regard to the (theoretical) concentration in the central compartment. This is mainly due to transportation delay as the drug concentration in the central compartment is not homogenous, which is evident especially during the transient response, i.e., when the amount of the drug in the central compartment is changing rapidly. The effect-site concentration is therefore a representation of a volumeless 4th compartment, where the drug is active. This compartment is virtually linked to the central compartment.

Therefore, a 1st order model has been used to describe the effect-site concentration dynamics, as given in

$$
\frac{d x_{e}}{d t}=-k_{e 0} x_{e}+k_{e 0} x_{1}
$$


where the virtual link between the central and the effectsite compartment is characterised by the coefficient $k_{e 0}$, which is actually the inverse time constant of the dynamic system describing the connection between the plasmatic concentration and the effect-site concentration $x_{e 0}$.

The schematics explaining the effect-site concentration dynamics are presented in in Figure 2.

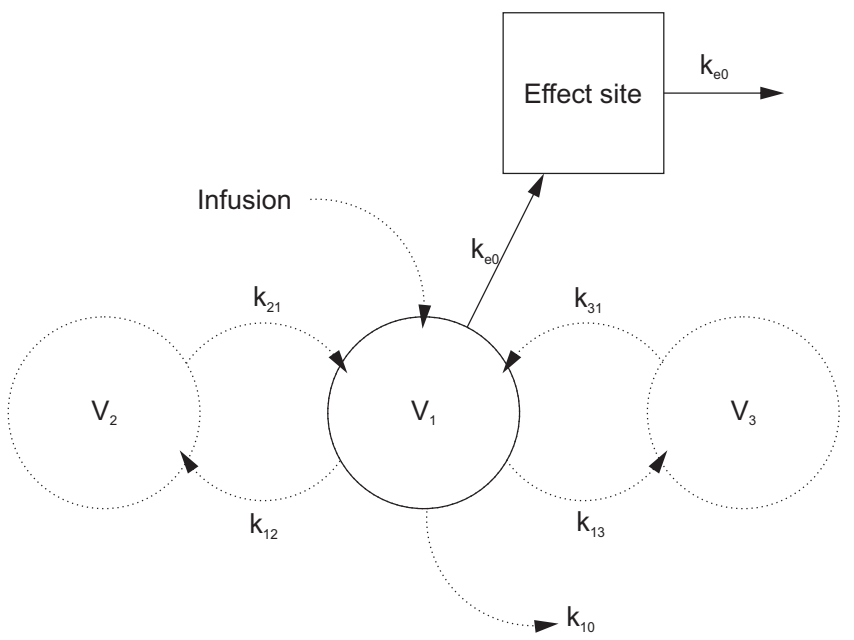

Figure 2. The effect-site concentration dynamics.

\subsection{Model parameters}

The parameters of the model are taken from (Marsh et al., 1991) and (Orc). The values are given in Table 1.

Table 1. Parameter values.

\begin{tabular}{|c|c|}
\hline Parameter & Value \\
\hline$V_{c}$ & $0.2281 / \mathrm{kg}$ \\
$k_{10}$ & $0.119 / \mathrm{min}$ \\
$k_{12}$ & $0.112 / \mathrm{min}$ \\
$k_{13}$ & $0.0419 / \mathrm{min}$ \\
$k_{21}$ & $0.055 / \mathrm{min}$ \\
$k_{31}$ & $0.0033 / \mathrm{min}$ \\
$k_{e 0}$ & $1.21 / \mathrm{min}$ \\
\hline
\end{tabular}

When developing the model in Matlab-Simulink we have to consider the units and the dilution factors of the drug. For example, the parameters expressed in $\mathrm{min}^{-1}$ have to be converted to $s^{-1}$. Furthermore, the input flow of propofol is typically expressed in $m l / h$, and the dilution is $10 \mathrm{mg} / \mathrm{ml}$, i.e., $1 \%$. The output concentration (plasmatic and effect-site) are expressed in $\mu \mathrm{g} / \mathrm{ml}$.

\section{Model verification}

The Marsh model is also used in the infusion workstation Orchestra Base Primea (produced by Fresenius Kabi) (Orc). We obtained a textual output data file of an actual anaesthetic application of target-controlled infusion of intravenously administered propofol, which was recorded by the Orchestra Base Primea infusion workstation during a medical procedure that lasted about 40 minutes. The data recorded in the output file are as follows:

- The infusion flow of propofol.

- The predicted plasmatic concentration of propofol $c_{p}$.

- The predicted effect-site concentration of propofol $c_{e}$.

- Important events (alarms, occlusions, syringe changes, target-value changes).

- Patient data:

- age (43 years),

- weight $(78 \mathrm{~kg})$,

- height $(177 \mathrm{~cm})$, and

- gender (male).

A Matlab-based parser was developed, which is able to processes the textual output data files recorded by the Orchestra Base Primea infusion workstation so as to obtain suitably formatted time-stamped data arrays. In such a manner, the obtained data arrays can easily be used in the Matlab-Simulink environment.

\section{Simulation results}

\subsection{Propofol inflow}

We used the presented model for simulating the system behaviour with regard to the response to the inflow of propofol influencing the depth of anaesthesia through plasmatic concentration and effect-site concentration of propofol. The simulated inflow of propofol was adjusted according to the data recorded by the Orchestra Base Primea infusion workstation.

The inflow-signal of propofol $\phi_{\text {propofol }}$ was parsed from the recorded data and is shown in Figure 3.

Note that first a bolus-dose is administered so as to rapidly increase the concentration of propofol in the body. This phase is called the induction of anaesthesia and results in the patient losing consciousness. Later, a suitable dose of propofol is continuously administered in order to keep the proper anaesthetic depth. A close-up of the second transient phase is shown in Figure 4.

The presented Matlab-Simulink model was fed the propofol-inflow signal $\phi_{\text {propofol }}$ and the resulting trajectories of propofol concentration in the central compartment and in the effect-site compartment $\left(c_{p}\right.$ and $c_{e}$, respectively) were compared to the data recorded by the Orchestra Base Primea infusion workstation. 


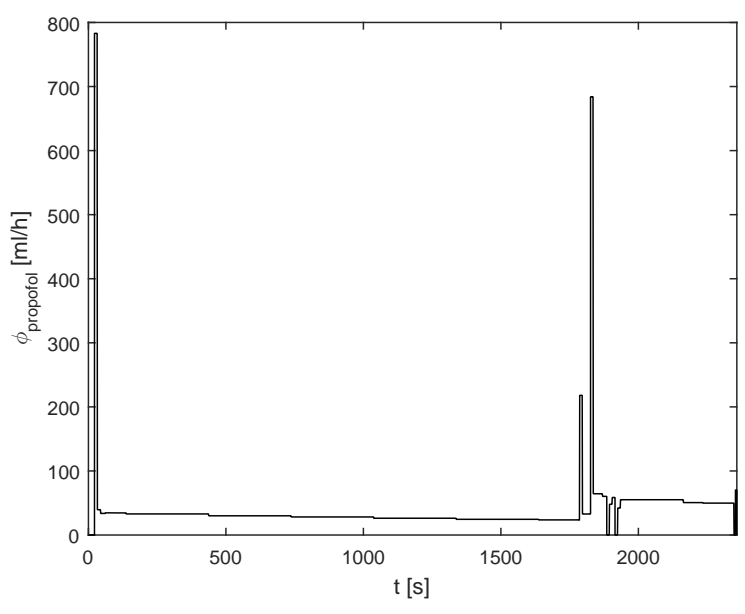

Figure 3. The inflow of propofol $\phi_{\text {propofol }}$.

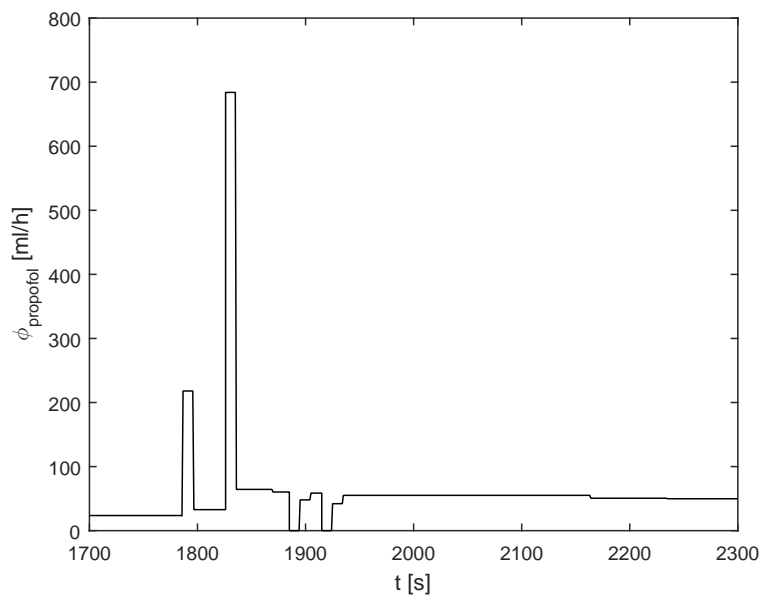

Figure 4. The inflow of propofol $\phi_{\text {propofol }}$ (close-up).

\subsection{Plasmatic concentration}

The simulated plasmatic concentration of propofol $c_{p, s i m}$ trajectory is shown in Figure 5. The simulated results are compared to the parsed data from the Orchestra Base Primea infusion workstation data file $c_{p, \text { data }}$. A close-up of the second transient phase is shown in Figure 6.

\subsection{Effect-site concentration}

Similarly, the simulated effect-site concentration of propofol $c_{e, s i m}$ trajectory is shown in Figure 7. The simulated results are compared to the parsed data from the Orchestra Base Primea infusion workstation data file $c_{e, \text { data. A }}$. A close-up of the second transient phase is shown in Figure 8.

\subsection{Evaluation of the predictive quality of the model}

In order to evaluate the predictive quality of the model, the simulated results are compared to the parsed data from the Orchestra Base Primea infusion workstation data file. Some established quantitative measures for predictive

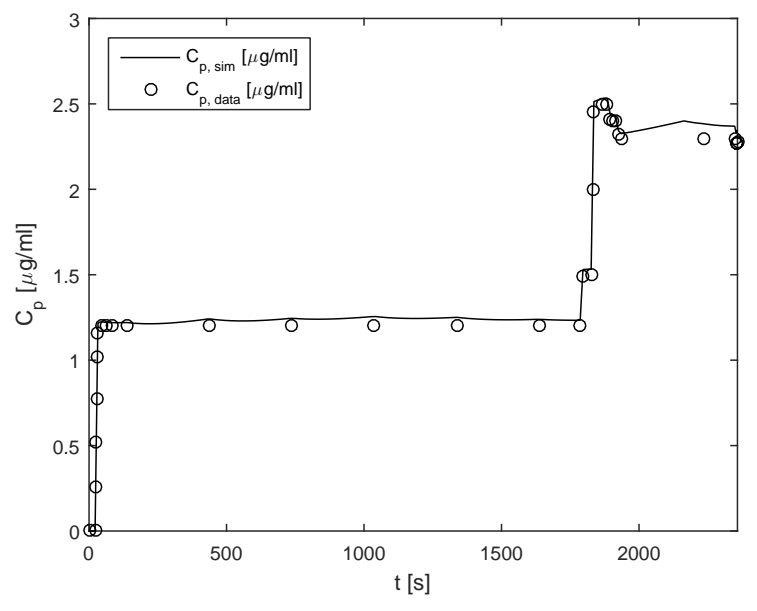

Figure 5. The simulated plasmatic concentration of propofol $c_{p, \text { sim }}$ compared to the parsed data $c_{p, \text { data }}$.

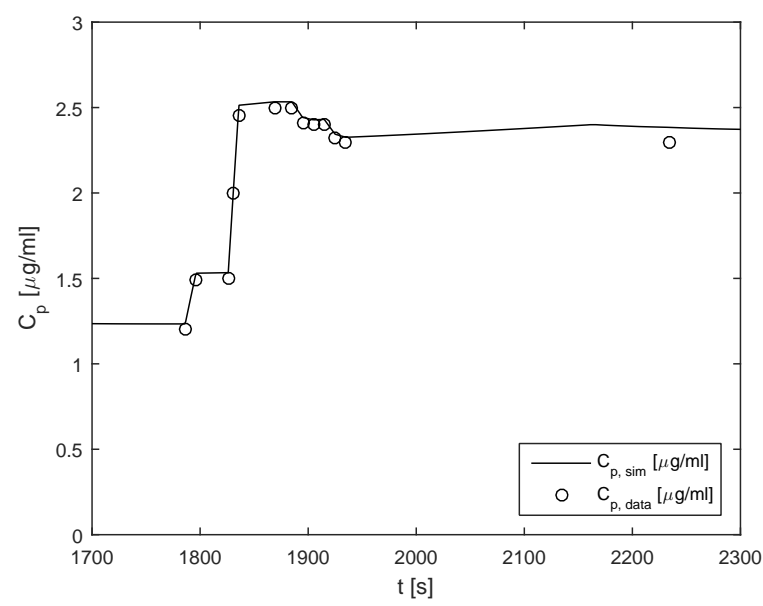

Figure 6. The simulated plasmatic concentration of propofol $c_{p, \text { sim }}$ compared to the parsed data $c_{p, \text { data }}$ (close-up).

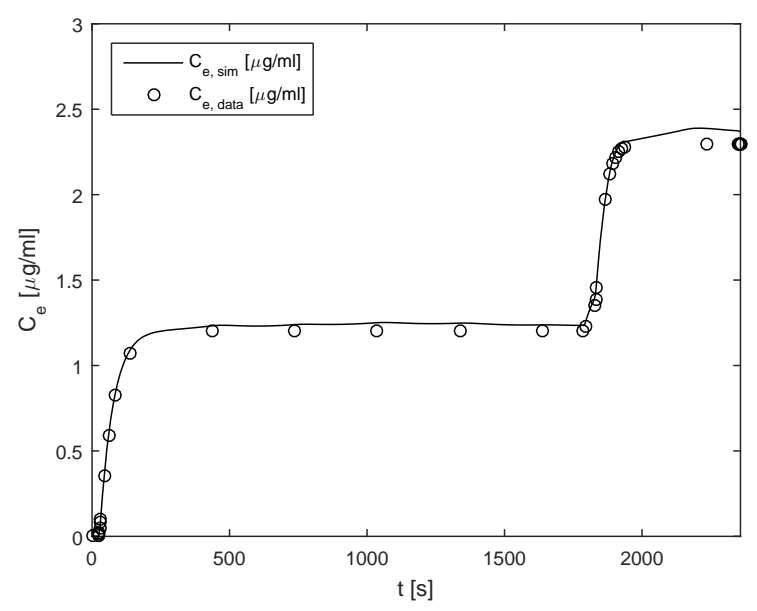

Figure 7. The simulated effect-site concentration of propofol $c_{e, \text { sim }}$ compared to the parsed data $c_{e, \text { data }}$. 


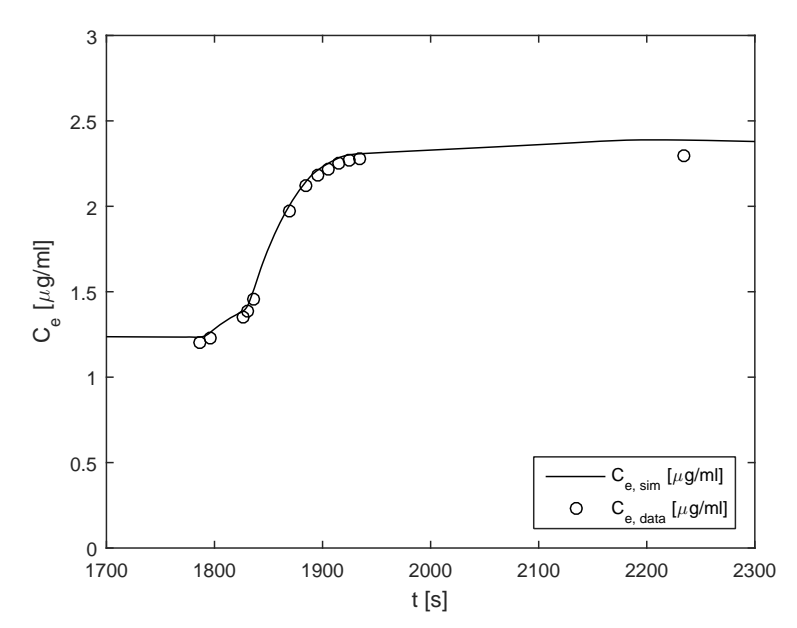

Figure 8. The simulated effect-site concentration of propofol $c_{e, s i m}$ compared to the parsed data $c_{e, \text { data }}$ (close-up).

quality are prediction mean square error (PMSE), median performance error (MDPE), and median absolute performance error (MDAPE) (Mertens et al., 2003). The aforementioned measures are calculated as defined in

$$
\begin{gathered}
\operatorname{PMSE}_{x}=\frac{1}{N} \sum_{i=1}^{N}\left(x_{i, \text { sim }}-x_{i, \text { data }}\right)^{2} \\
M D P E_{x}=\text { median }\left\{\frac{x_{i, \text { data }}-x_{i, \text { sim }}}{x_{i, \text { sim }}} \cdot 100 \%\right\}_{i=1, \ldots, N} \\
M D A P E_{x}=\operatorname{median}\left\{\left|\frac{x_{i, \text { data }}-x_{i, \text { sim }}}{x_{i, \text { sim }}}\right| \cdot 100 \%\right\}_{i=1, \ldots, N}
\end{gathered}
$$

where $x_{\text {sim }}$ and $x_{\text {data }}$ stand for the simulated and the "measured" data, respectively, and $N$ is the number of data points in the data set.

In our case, the values of the aforementioned measures are presented in Table 2 .

Table 2. Predictive quality measures.

\begin{tabular}{|c|c|c|c|}
\hline Signal $x$ & $P M S E_{x}$ & $M D P E_{x}$ & $M D A P E_{x}$ \\
\hline$C_{p}$ & $2.21 \cdot 10^{-3}$ & $-1.67 \%$ & $2.00 \%$ \\
$C_{e}$ & $1.62 \cdot 10^{-3}$ & $-2.35 \%$ & $2.78 \%$ \\
\hline
\end{tabular}

As the Orchestra Base Primea infusion workstation suffers from some error when logging the propofol-flow data $\phi_{\text {propofol }}$, it is sensible to take into account the final cumulative amount of the administered drug. In this case, the total amount of propofol used was $25.5 \mathrm{ml}$, whereas the simulated consumption was $26.2 \mathrm{ml}$. It is clear that the simulated propofol concentration signals, both plasmatic and effect-site, are influenced considerably by the aforementioned error.

In general, the propofol-flow $\phi_{\text {propofol }}$ error is generally not uniformly distributed. Nevertheless, the simulation results can be improved by simply pondering the simulated propofol inflow by a factor of $\frac{25.5 \mathrm{ml}}{26.2 \mathrm{ml}}$. The newly simulated results are again compared to the parsed data from the Orchestra Base Primea infusion workstation data file in Figures 9 and 10 for plasmatic and effect-site concentration trajectories, respectively.

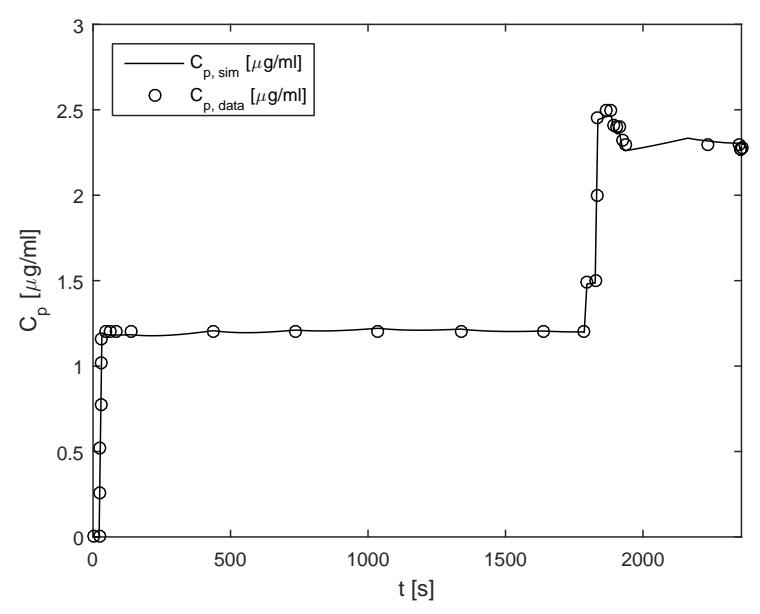

Figure 9. The newly simulated plasmatic concentration of propofol $c_{p, s i m}$ compared to the parsed data $c_{p, \text { data }}$.

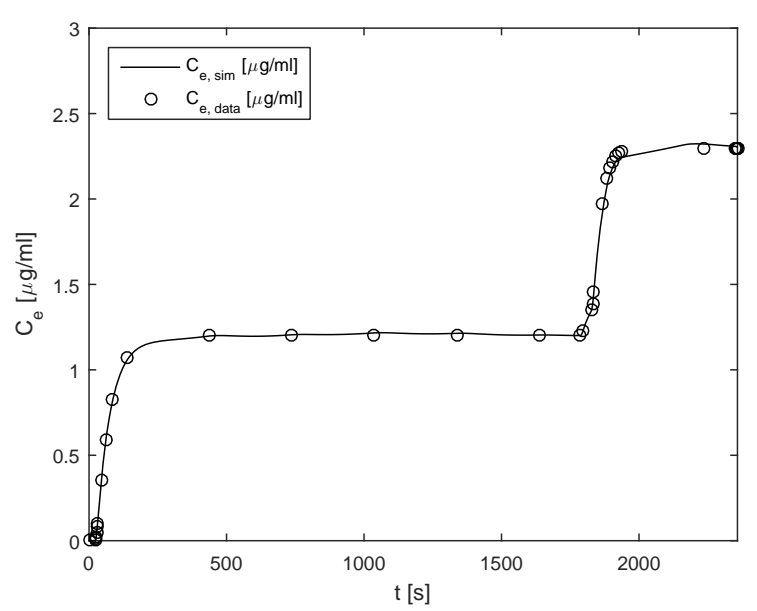

Figure 10. The newly simulated effect-site concentration of propofol $c_{e, s i m}$ compared to the parsed data $c_{e, \text { data }}$.

In this manner, the predictive quality measures are improved by a considerable amount, as shown in Table 3 .

Table 3. Predictive quality measures (modified).

\begin{tabular}{|c|c|c|c|}
\hline Signal $x$ & $P M S E_{x}$ & $M D P E_{x}$ & $M D A P E_{x}$ \\
\hline$C_{p}$ & $0.65 \cdot 10^{-3}$ & $1.16 \%$ & $1.38 \%$ \\
$C_{e}$ & $0.32 \cdot 10^{-3}$ & $0.324 \%$ & $1.14 \%$ \\
\hline
\end{tabular}

\section{Conclusions}

The developed model for Matlab-Simulink provides a basic tool for further researching the dynamics of depth of 
anaesthesia. The model enables improvement and refinement of model quality for propofol for assessment of the dynamic properties of anaesthetic depth. However, more data must be obtained in order to properly validate the model. We will work with the anaesthesiologic team of the University clinical centre in Ljubljana in order to reach this goal. In addition, the measured data from real medical procedures will enable further refinement of the model parameters and possibly a structural improvement of the considered dynamical relations.

That said, the presented model provides a basis for running simulations and testing various scenarios of propofol administration that could lead to better administration protocols due to a deeper insight of the mechanisms of depth of anaesthesia.

Finally, the model is usable for developing and testing closed-loop control approaches for automatic control of anaesthetic depth, which will be the main direction of our joint future research in collaboration with the anaesthesiologic team of the University clinical centre in Ljubljana.

\section{References}

Operator's Guide: Infusion Workstation: Orchestra Base Primea.

B. K. Kataria, S. A. Ved, H. F. Nicodemus, G. R. Hoy, D. Lea, M. Y. Dubois, J. W. Mandema, and S. L. Shafer. The pharmacokinetics of propofol in children using three different data analysis approaches. Anesthesiology, 80:104-122, 1994.

G. N. Kenny and M. White. Intravenous propofol anaesthesia using a computerised infusion system. Anaesthesia, 46:204209, 1990.

B. Marsh, M. White, N. Morton, and G. N. Kenny. Pharmacokinetic model driven infusion of propofol in children. $\mathrm{Br} \mathrm{J}$ Anaesth, 67:41-48, 1991.

M. J. Mertens, F. H. M. Engbers, A. G. L. Burm, and J. Vuyk. Predictive performance of computer-controlled infusion of remifentanil during propofol/remifentanil anaesthesia. $\mathrm{Br} \mathrm{J}$ Anaesth, 90(2):132-141, 2003.

I. Potočnik, V. Novak Janković, T Štupnik, and B. Kremžar. Haemodynamic changes after induction of anaesthesia with sevoflurane vs. propofol. Signa Vitae, 6(2):52-57, 2011.

T. W. Schnider, C. F. Minto, P. L. Gambus, C. Andresen, D. B. Goodale, S. L. Shafer, and E. J. Youngs. The influence of method of administration and covariates on the pharmacokinetics of propofol in adult volunteers. Anesthesiology, 88: 1170-1182, 1998.

T. W. Schnider, C. F. Minto, S. L. Shafer, P. L. Gambus, C. Andresen, D. B. Goodale, and E. J. Youngs. The influence of age on propofol pharmacodynamics. Anesthesiology, 90:1502$1516,1999$.

J. Schüttler and H. Ihmsen. Population pharmacokinetics of propofol: a multicenter study. Anesthesiology, 92(3):727738, 2000. 